\title{
Antagonists of Cyclic Nucleotide-Gated Channels and Molecular Mapping of their Site of Action
}

\author{
Richard H. Kramer ${ }^{1}$ and Gareth R. Tibbs ${ }^{2}$ \\ ${ }_{1}^{1}$ Department of Molecular and Cellular Pharmacology, University of Miami School of Medicine, Miami, Florida 33101 , \\ and ${ }^{2}$ Center for Neurobiology and Behavior, Columbia University School of Physicians and Surgeons, New York, \\ New York 10032
}

Activation of photoreceptor and olfactory cyclic nucleotidegated (CNG) channels involves distinct ligand-binding and channel-gating reactions. To dissociate binding from gating, we identified the first competitive antagonists of CNG channels: specific phosphorothioate derivatives of cAMP and cGMP. We also identified membrane-permeant forms of these molecules that are antagonists and that will be useful for elucidating physiological roles for CNG channels in intact cells. The photoreceptor and olfactory CNG channels determine which of the phosphorothioate derivatives are agonists and which are antagonists based on different structural features of the ligand. The photoreceptor channel uses the nature of the purine ring (adenine vs guanine), whereas the olfactory channel uses the isomeric position of the thiophosphate $\mathrm{S}$ atom (Rp vs $\mathrm{Sp}$ ). Interestingly, the same ligand, Rp-cGMPS, has opposite effects on the two channels, activating the photoreceptor channel and antagonizing the olfactory channel. Because Rp-cGMPS binds to both channels but activates only one, the channels must differ in a protein region that couples binding to gating. Chimeric photoreceptor and olfactory CNG channels reveal that the cytoplasmic C-terminal domain determines whether bound ligand activates the channel successfully. Hence, the $C$ terminus contains not only the cyclic nucleotide-binding site, but also a region that couples ligand binding to channel gating.

Key words: ligand-gated channels, CAMP, cGMP, photoreceptors, olfactory neurons, cyclic nucleotide-binding proteins
Cyclic nucleotide-gated (CNG) channels are crucial components of the sensory transduction process in retinal photoreceptors and olfactory receptor neurons. During sensory stimulation, the opening or closing of these channels acts to transduce a change in the intracellular cyclic nucleotide concentration into an electrical signal. The CNG channels differ in that cAMP and cGMP are nearly equally effective in activating olfactory CNG channels (Nakamura and Gold, 1987; Dhallan et al., 1990; Goulding et al., 1992; Goulding et al., 1994), whereas activation of photoreceptor CNG channels is highly selective for cGMP (Fesenko et al., 1985; Kaupp et al., 1989). Recently, CNG channels have been found in a surprising assortment of other cells (Yau et al., 1994), including retinal neurons (Nawy and Jahr, 1991; Ahmad et al., 1994), cardiac myocytes (DiFrancesco et al., 1991; Biel et al., 1994), and sperm (Weyand et al., 1994). Membrane-permeant cyclic nucleotide analogs that are agonists have been useful for implicating the presence and identifying the functions of CNG channels in these cells. However, competitive antagonists of CNG channels have not yet been identified.

To identify antagonists of CNG channels, we examined molecules known to antagonize cyclic nucleotide-dependent protein kinases. The recent cloning of genes encoding the rod photoreceptor (Kaupp et al., 1989; Chen et al., 1993) and olfactory CNG

\footnotetext{
Received Aug. 28, 1995; revised Nov. 1, 1995; accepted Nov. 7, 1995.

This work was supportcd by National Institutes of Hcalth Grant NS30695. We thank Charles Luetje for help with oocyte expression, Steven Siegelbaum for encouragement and support, and Beth Arnitage and Ellen Barrett for insightful comments on this manuscript. We also thank Darren Mitchell and John Riley for excellent technical support and Chuck Lam for help with figures.

Correspondence should be addressed to Dr. Richard H. Kramer, Department of Molecular and Cellular Pharmacology, P.O. Box 016189, University of Miami School of Medicine, Miami, FL 33101.

Copyright $(1996$ Society for Neuroscience $0270-6474 / 96 / 161285-09 \$ 05.00 / 0$
}

channels (Dhallan et al., 1990; Goulding et al., 1992) has revealed a conserved region in the cytoplasmic C-terminal domain that is highly homologous to the cyclic nucleotide-binding domains of cAMP-dependent protein kinase (PKA) and cGMP-dependent protein kinase (PKG). Specific phosphorothioate derivatives of CAMP and CGMP inhibit the kinases competitively, with RpcAMPS inhibiting PKA (Botelho et al., 1988) and Rp-cGMPS inhibiting PKG (Butt et al., 1990). Here, we show that these phosphorothioate derivatives also antagonize CNG channels. In addition, we show that derivatives of these molecules containing a $p$-chlorophenylthio (pCPT) group at the 8-position of the purine are higher-affinity antagonists with the advantage of being highly membrane-permeant. Hence, these compounds may be useful tools for assessing physiological roles of CNG channels in various cells.

We have observed that some phosphorothioate derivatives have strikingly different effects on two classes of CNG channels. Thus, $\mathrm{Rp}$-cGMPS is an agonist of photoreceptor CNG channels and an antagonist of olfactory $\mathrm{CNG}$ channels. We have used this observation to address the molecular mechanism of CNG channel activation. Activation of $\mathrm{CNG}$ channels is a multistep process involving distinct ligand-binding and channel-opening steps. Competitive antagonists bind without opening the channel and, therefore, dissociate these processes. Since the same ligand binds to both channels but selectively activates only one, the two CNG channel proteins must differ in a region that couples ligand binding to channel opening. To identify this region, we examined effects of the phosphorothioates on chimeric combinations between the two CNG channels. Our results show that the cytoplasmic C-terminal domain of $\mathrm{CNG}$ channels not only contains the cyclic nucleotide-binding site, but also contains a region that couples ligand binding to channel opening. 


\section{MATERIALS AND METHODS}

Electrophysiological recording and analysis. Patch electrodes (tip diameter, 1-3 $\mu \mathrm{m})$ were fabricated from Kimax-51 borosilicate glass. For singlechannel recording experiments, the electrodes were coated with Sylgard to reduce capacitance. CNG currents were monitored with an EPC-7 (List, Campbell, CA) or an Axopatch 200A (Axon Instruments, Foster City, CA) patch-clamp amplifier. Macroscopic current data were filtered at $1 \mathrm{kHz}$ and stored on videotape for later computer analysis. Singlechannel data were filtered at $4 \mathrm{kHz}$, stored on videotape, and digitized later at $20 \mathrm{kHz}$ with a Macintosh Centris 650 computer (Pulse software, Instrutech, Great Neck, NY). Single-channel conductance was determined from Gaussian fits to all points amplitude histograms with programs written in Axobasic (Axon Instruments). Burst analysis was performed on the same data using MacTAC (Skalar Instruments). Variability in the data is expressed as mean $\pm \mathrm{SEM}$. All experiments were done at room temperature $\left(20-24^{\circ} \mathrm{C}\right)$. Solutions were superfused continuously over the cytoplasmic surface of excised patches at $1-2 \mathrm{ml} / \mathrm{min}$. Rapid solution changes were made with a 15-channel microperfusion system. Current responses to cyclic nucleotide applications were normalized to the maximal CNG current $\left(I_{\max }\right)$ elicited by a saturating concentration of cGMP (1-10 mM cGMP) and fit to the Hill equation: $I / I_{\max }=$ $1 /\left(1+\left(K_{1 / 2} /[A]\right)^{n}\right)$, where $A$ is the cyclic nucleotide concentration and $n$ is the Hill coefficient.

Recording from native olfactory CNG channels. Olfactory CNG channels were studied in excised "macro" patches containing the apical dendrite and receptor cilia of olfactory receptor neurons (Kramer and Siegelbaum, 1992). Olfactory neurons were obtained from the channel catfish (Ictalurus punctatus). All animals used in this study were maintained and used according to NIH guidelines. Olfactory neurons were acutely dissociated and stored at $4^{\circ} \mathrm{C}$ in a solution containing (in $\mathrm{mm}$ ): $\mathrm{NaCl} 110, \mathrm{KCl} 3$ $\mathrm{MgCl}_{2} 2, \mathrm{CaCl}_{2}$ 1, glucose 5, and HEPES 10, pH 7.5, as described previously (Kramer and Siegelbaum, 1992). Both the patch pipettes and the superfusion solutions contained (in mM): $\mathrm{NaCl} \mathrm{100,} \mathrm{EGTA} \mathrm{1,} \mathrm{and}$ HEPES 10, pH 7.2. Maximal CNG currents in excised patches ranged from $50 \mathrm{pA}$ to $2.0 \mathrm{nA}$ for olfactory neurons, $500 \mathrm{pA}$ to $2.5 \mathrm{nA}$ for rod photoreceptors, and $25 \mathrm{pA}$ to $5 \mathrm{nA}$ for oocytes. Large CNG currents that exhibited "droop" because of ion accumulation (Zimmerman et al., 1988) such that the CNG current declined by $>5 \%$ during cyclic nucleotide application were excluded from consideration in this paper.

Recording from native rod photoreceptor $C N G$ channels. Photoreceptor CNG channels were studied in excised patches from mechanically dissociated intact rods or rod outer segments from dark-adapted tiger salamanders (Ambystoma tigranum). Salamanders were killed by decapitation, and the retina was removed and triturated gently in saline of the same composition as described above. Both the patch pipettes and the superfusion solutions contained (in $\mathrm{mm}$ ): $\mathrm{NaCl} 115$, EGTA 5, and HEPES 5, $\mathrm{pH} 7.5$. Isobutylmethylxanthine $(100 \mu \mathrm{M})$ was included in all perfusion solutions to block phosphodiesterase activity. CNG currents in excised patches from rods exposed to these solutions exhibited no spontaneous change in sensitivity to cyclic nucleotides for at lcast $30 \mathrm{~min}$ after excision (Gordon et al., 1992).

Recording from expressed CNG channels. Cloned bovine rod photoreceptor (NG channels (RET channels) (Kaupp et al., 1989), cloned catfish olfactory receptor neuron CNG channels (OLF channels) (Goulding et al., 1992), and chimeric (Goulding et al., 1994) CNG channels were used. The new RON-S2-BD chimera was constructed by substituting the OLF cyclic nucleotide-binding domain (amino acids $455 \mathrm{~L}$ to $580 \mathrm{E}$ from chimera ROCB) (Goulding et al., 1994) into the corresponding position of RON-S2 (amino acids 485L to 610E) using restriction sites shared by the two sequences. Channels were expressed in Xenopus oocytes as described previously (Kramer and Siegelbaum, 1992). Expcriments wcre performed 1-7 d after mRNA injection. Before patch clamping, oocytes were incubated in hyperosmotic stripping solution containing (in $\mathrm{mM}$ ): potassium aspartate $200, \mathrm{KCl} 20, \mathrm{MgCl}_{2} 1$, and HEPES $10, \mathrm{pH} 7.4$; the vitelline membrane was then removed.

Cyclic nucleotides. Phosphorothioate cAMP and cGMP derivatives were obtained from BioLog (La Jolla, CA). These include (Rp)- and (Sp)-adenosine cyclic $3^{\prime}, 5^{\prime}$-phosphorothioate (Rp-cAMPS and SpcAMPS), (Rp)- and (Sp)-guanosine cyclic $3^{\prime}, 5^{\prime}$-phosphorothinate (Rp-cGMPS and Sp-cGMPS), (Rp)-8-p-chlorophenylthioadenosine3',5'-phosphorothioate (Rp-8-pCPT-cAMPS), and (Rp)-8-p-chlorophenylthioguanosine-3',5'-phosphorothioatc (Rp-8-pCPT-cGMPS) cAMP and cGMP were obtained from Sigma (St. Louis, MO). Stock solutions of cyclic nucleotides $(10-50 \mathrm{~mm})$ were stored in water or

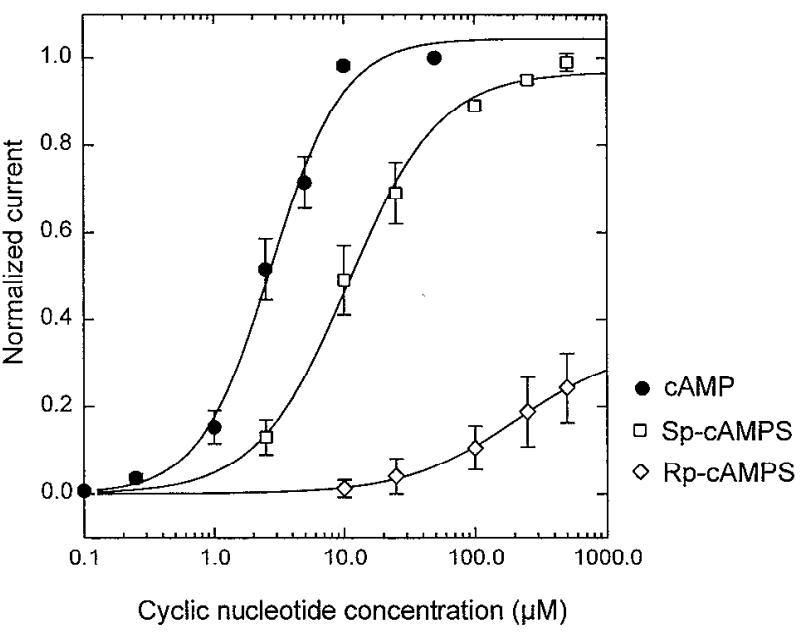

Figure 1. Activation of olfactory $\mathrm{CNG}$ channels by phosphorothioate cyclic AMP derivatives. Dose-response curves show magnitude of CNG current in excised patches from catfish olfactory neurons. Responses in each patch were normalized to the response elicited by a saturating dose of cAMP $(1000 \mu \mathrm{M})$, for cAMP (filled circles; $N=12$ ), Sp-cAMPS (open squares; $N=3$ ), and Rp-cAMPS (open diamonds; $N=4$ ). Solid lines represent best fits to the Hill equation with values of $K_{1 / 2}, n$ (Hill coefficient), and $I_{\max }$ of: $2.8 \mu \mathrm{M}, 1.6$, and 1.04 for cAMP; $10.8 \mu \mathrm{M}, 1.2$, and 0.97 for Sp-cAMPS; and $207 \mu \mathrm{M}, 1.0$, and 0.34 for Rp-cAMPS.

patch saline at $-20^{\circ} \mathrm{C}$. Working solutions containing different concentrations were used within $24 \mathrm{hr}$ of preparation.

We were concerned that our stock of Rp-cAMPS may have become desulfonated over time, generating cAMP and possibly accounting for our observation that Rp-cAMPS can be a partial agonist of olfactory CNG channels. Tests of Rp-cAMPS samples using reverse-phase highperformance liquid chromatography with an RP-C18 column (Bondapak) equilibrated in $1 \mathrm{mM} \mathrm{Na}_{3} \mathrm{PO}_{4}$ and $1 \%$ methanol, $\mathrm{pH} 6.5$, showed that samples contained $<0.5 \%$ cAMP. At the highest concentration of RpcAMPS used on the native olfactory CNG channel $(500 \mu \mathrm{M})$, this would be equivalent to $2.5 \mu \mathrm{M}$ cAMP. To eliminate possible contamination of Rp-cAMPS by cAMP, we treated samples of Rp-cAMPS with phosphodiesterase (PDE) from the slime mold Dictyostelium discoideum. This PDE hydrolyzes cAMP without hydrolyzing or being inhibited by Rp-cAMPS (R. Kessin, personal communication). Parallel control samples containing radiolabeled cAMP ([8- $\left.{ }^{3} \mathrm{H}\right]$ cAMP; Amersham, Arlington Heights, IL) along with Rp-cAMPS also were treated and analyzed by ascending paper chromatography. After treatment sufficient to reduce cAMP to $0.01 \%$ of its initial level, the label-free Rp-cAMPS sample was tested on native olfactory CNG channels. The PDE-treated Rp-cAMPS activated olfactory CNG channels to the same extent as untreated Rp-cAMPS, indicating that agonist activity could not have been caused by cAMP contamination.

\section{RESULTS}

\section{Rp-cAMPS is a partial agonist of the olfactory CNG channel}

Figure 1 plots the effect of phosphorothioate isomers of cAMP on CNG channels in excised patches from dissociated catfish olfactory neurons. Activation curves were normalized to the peak response elicited by a saturating concentration of cAMP (1 mM), and averaged data were fitted to the Hill equation. Sp-cAMPS, like cAMP, is a full agonist of the olfactory CNG channel, but has a lower apparent affinity $\left(K_{1 / 2}=2.8 \mu \mathrm{M}\right.$ for $\mathrm{cAMP}$ and $11 \mu \mathrm{M}$ for Sp-cAMPS). In contrast, Rp-cAMPS does not appear to be a full agonist. Even at high concentrations approaching saturation, this analog activates $<35 \%$ of the maximal current activated by a saturating concentration of cAMP. Rp-cAMPS also has a weaker apparent binding affinity to the channels, with an estimated $K_{1 / 2}$ of $207 \mu \mathrm{M}$. 
A

$2.5 \mu \mathrm{M} \mathrm{cAMP}$

$200 \mu \mathrm{M}$ Rp-cAMPS
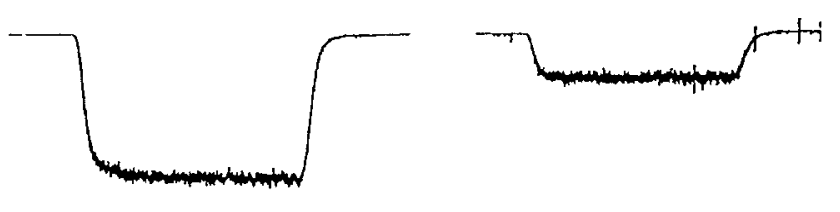

B

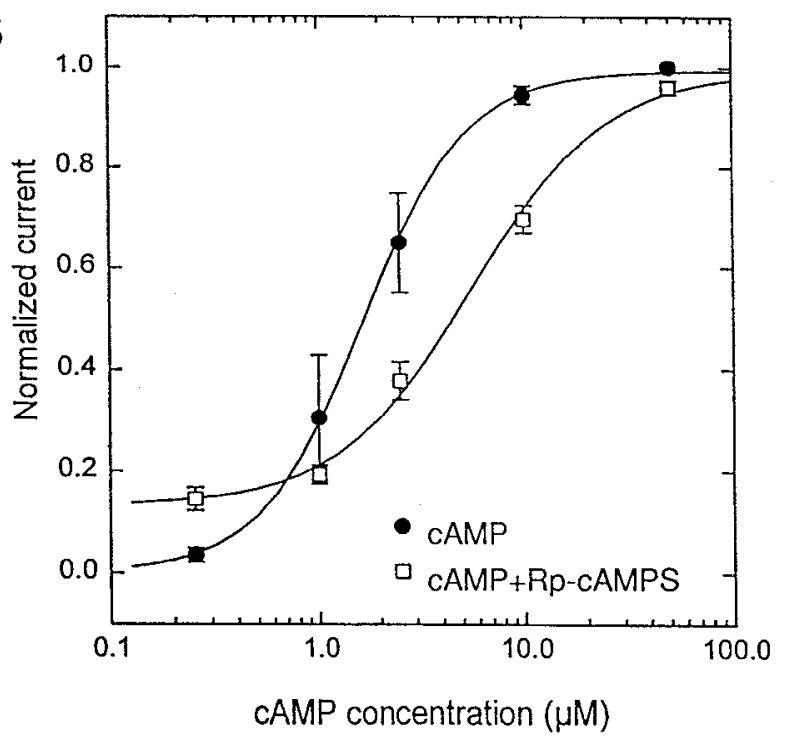

$200 \mu \mathrm{M}$ Rp-CAMPS $+2.5 \mu \mathrm{M}$ сAMP

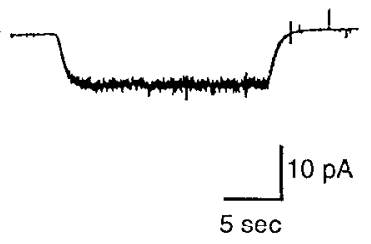

Figure 2. Antagonism of cAMP responses in olfactory $\mathrm{CNG}$ channels by Rp-cAMPS. $A$, Currents resulting from application of $C A M P$, $R p-c A M P S$, or Rp-cAMPS + cAMP. Holding potential, $-50 \mathrm{mV} . B$, Dose-response curves of CNG current elicited by cAMP alone (filled circles) or cAMP $+500 \mu \mathrm{M}$ Rp-cAMPS (open squares) for five measurements each. Solid curves show Hill fits to the data with a $K_{1 / 2}$ of $1.7 \mu \mathrm{M}$ for cAMP alone and $5.7 \mu \mathrm{M}$ in the presence of Rp-cAMPS.

If Rp-cAMPS occupies the usual binding site without fully activating the channel, it should compete with more effective ligands, such as cAMP and cGMP. Figure $2 A$ shows CNG current from native olfactory $\mathrm{CNG}$ channels elicited by cAMP and Rp-cAMPS and the two nucleotides in combination. Not only are the responses not additive, but Rp-cAMPS inhibits the current that would have been elicited by cAMP alone. The interaction betwecn Rp-cAMPS and cAMP indecd is competitive, with Rp-cAMPS inhibiting cAMP-elicited current only at subsaturating cAMP concentrations, as shown in the doseresponse curves in Figure $2 B$. Thus, responses elicited by 2.5 or $10 \mu \mathrm{M}$ cAMP are inhibited, whereas responses to $50 \mu \mathrm{M}$ cAMP are not. Rp-cAMPS also inhibits current elicited by cGMP competitively (data not shown).

\section{Rp-cAMPS is an antagonist of the photoreceptor CNG channel}

Rp-cAMPS appears to be a pure antagonist of CNG channels from outer segments of salamander rod photoreceptors. RpcAMPS, even at $2 \mathrm{~mm}$, elicits no detectable $\mathrm{CNG}$ current in excised patches from rod outer segments (data not shown), but inhibits the response to subsaturating concentrations of cGMP competitively (e.g., $10-50 \mu \mathrm{M}$ ) by as much as $95 \%$ (Fig. $3 A$ ). Rp-cAMPS has no effect on current elicited by maximal concentrations ( $1 \mathrm{~mm}$ ) of cGMP (Fig. $3 A$ ). Rp-cAMPS acts as a classic competitive antagonist; in six experiments using cloned photoreceptor channels expressed in oocytes, $500 \mu \mathrm{M}$ Rp-cAMPS shifted the mean $K_{1 / 2}$ for cGMP activation from 68.7 to $106 \mu \mathrm{M}$ without altering the shape of the dose-response relationship or the maximal response (Fig. 3B).

\section{Rp-8-pCPT cAMPS is a higher-affinity antagonist of photoreceptor CNG channels}

Photoreceptor $\mathrm{CNG}$ channels are activated by 8 -thio-substituted analogs of cGMP and have a higher apparent affinity for these analogs than for cGMP itself (Brown ct al., 1993). Likewise, 8-pCPT-cAMP has a higher apparent affinity than cAMP for the olfactory CNG channel (our unpublished observations). These observations suggested that the pCPT form of Rp-cAMPS (Rp8-pCPT-cAMPS) might be a higher-affinity antagonist for the photoreceptor CNG channel than Rp-cAMPS. Indeed, we find that Rp-8-pCPT-cAMPS activates no current when applied alone, but antagonizes activation of photoreceptor CNG chamels strongly when applied together-with cGMP (Fig. 4A). Rp-cAMPS exhibits an $\mathrm{IC}_{50}$ of $435 \mu \mathrm{M}$, whereas Rp-8-pCPT-cAMPS exhibits an $\mathrm{IC}_{50}$ of $199 \mu \mathrm{M}$ (Fig. $4 B$ ).

\section{Rp-cAMPS alters gating of CNG channels}

Rp-cAMPS could inhibit the olfactory CNG current by reducing the number, single-channel conductance, and/or open probability of individual $\mathrm{CNG}$ channels. Figure $5 A$ shows a recording from an oocyte patch containing only a single OLF CNG channel. Application of Rp-cAMPS increases the interval between bursts of channel npenings and decreases the duration of these bursts, causing a decrease in open probability. RpcAMPS does not alter the number of active channels in the 
A
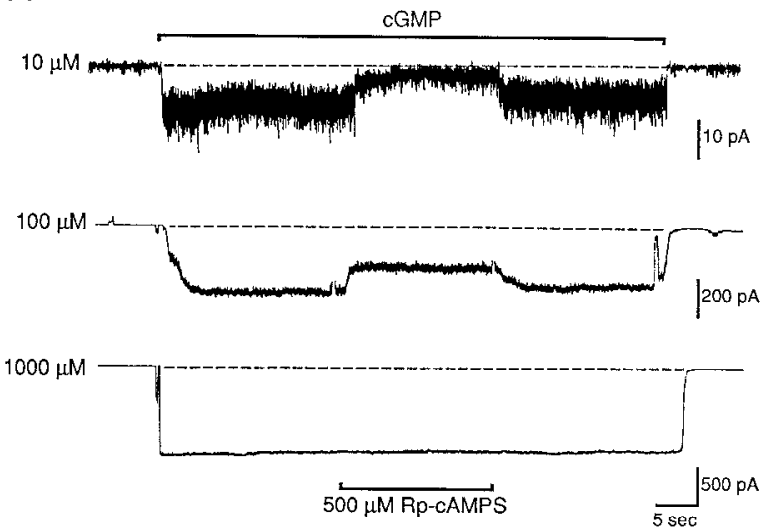

B

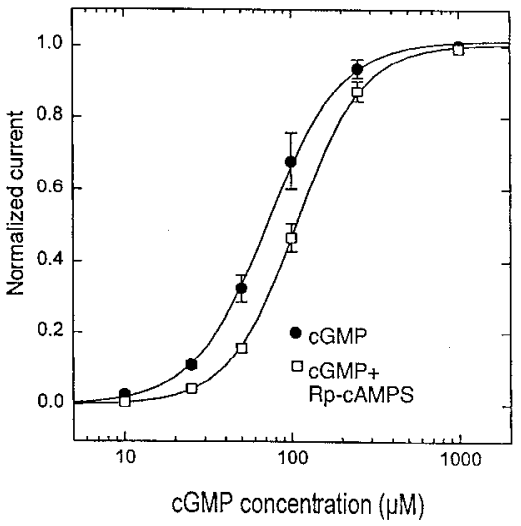

Figure 3. Antagonism of cGMP responses in rod photoreceptor CNG channels by Rp-cAMPS. $A$, Application of $500 \mu \mathrm{M}$ Rp-cAMPS antagonizes channel activation elicited by 10 and $100 \mu \mathrm{M}$ cGMP, but has no effect on the response elicited by a saturating concentration of cGMP $(1000 \mu \mathrm{M})$. $B$, Dose-response curves of cGMP activation of the cloned RET channel expressed in oocytes in the absence (filled circles) and presence (open squares) of $500 \mu \mathrm{M}$ Rp-cAMPS for six measurements each. Solid curves show Hill fits with $K_{1 / 2}, n$, and $I_{\max }$ of $71 \mu \mathrm{M}, 2.1$, and 1.00 with cAMP alone and $106 \mu \mathrm{M}, 2.2$, and 1.00 in the presence of Rp-cAMPS, respectively.

patch, nor does it alter single-channel conductance $(56.7 \pm 2.1$ $\mathrm{pS}$ before and $57.3 \pm 2.2 \mathrm{pS}$ after adding Rp-cAMPS; $n=4$ ). The amplitude of a subconductance state observed when single-channel events are recorded with high bandwidth also is unaltered by Rp-cAMPS $(29.7 \pm 1.4 \mathrm{pS}$ before and $29.7 \pm 1.5$ pS after Rp-cAMPS; $n=7$ ). Kinetic analysis reveals that Rp-cAMPS greatly increases the time constant of the major component of the closed time distribution from 9.3 to 21.2 msec. Figure $5 B$ shows that the open time distribution can be fit with three exponential components $(\tau=0.3,6.5$, and 38.4 msec). Rp-cAMPS decreases the proportion of long-lived events such that the open time distribution can now be fit with two components $(\tau=0.3$ and $4.5 \mathrm{msec})$. The observation that Rp-cAMPS decreases burst duration is consistent with the hypothesis that CNG channels can exist in more than one open state, with occupancy of the different open states related to the number of agonist molecules bound (Matthews and Watanabe, 1987). This model predicts that channels that are multiply occupied by agonists will dwell longer in open states and, conversely, that in the presence of an antagonist, the channel will spend less time in these long-lived open states.
A
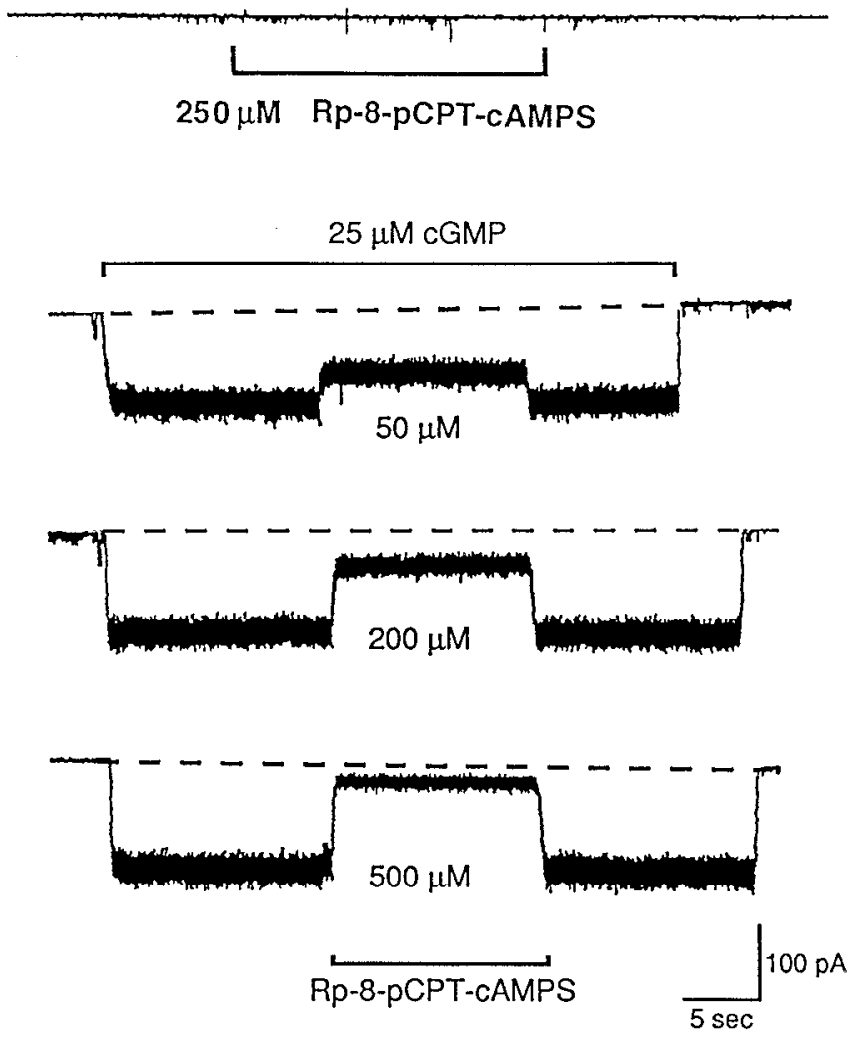

B

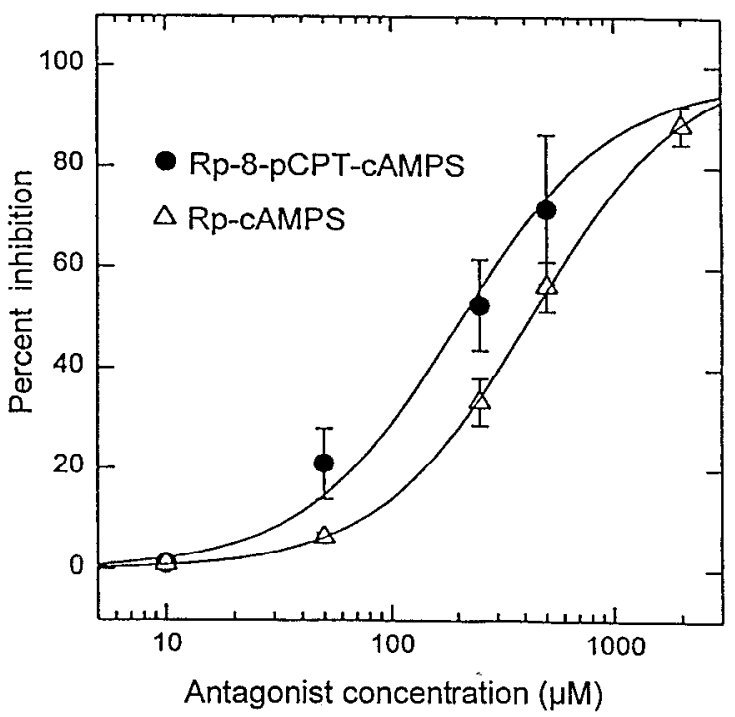

Figure 4. Antagonism of cGMP responses in rod photoreceptor CNG channels by Rp-8-pCPT-cAMPS. $A$, Application of $250 \mu \mathrm{M}$ Rp-8-pCPTcAMPS alone fails to activate rod $\mathrm{CNG}$ channels. Increasing concentrations of Rp-8-pCPT-cAMPS $(50,200$, and $500 \mu \mathrm{M})$ inhibit an increasing fraction of CNG current elicited by cGMP. cGMP $(25 \mu \mathrm{M})$ was near the $K_{1 / 2}$ for activating the CNG channels in this patch. $B$, Comparison between the effect of Rp-cAMPS (open triangles) and Rp-8pCPT-cAMPS (filled circles) on native rod CNG channels. Data represent mean $\pm \mathrm{SEM}$ for six measurements. The estimated $\mathrm{IC}_{50}$ for antagonism of CNG channels was $435 \mu \mathrm{M}$ for Rp-cAMPS and $199 \mu \mathrm{M}$ for Rp-8-pCPT-cAMPS. 
A

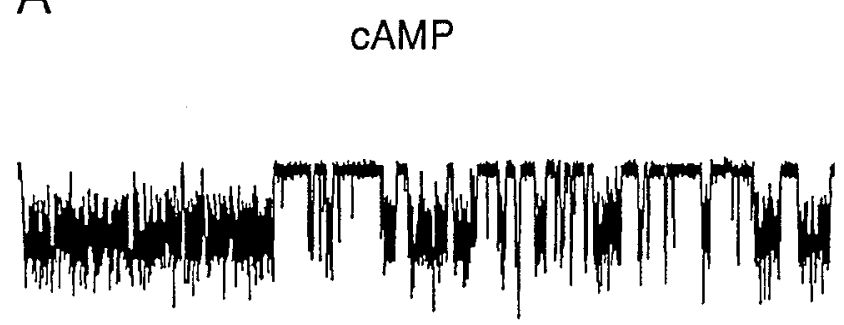

cAMP + Rp-cAMPS

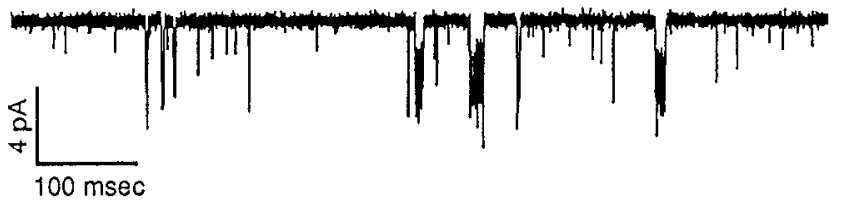

B

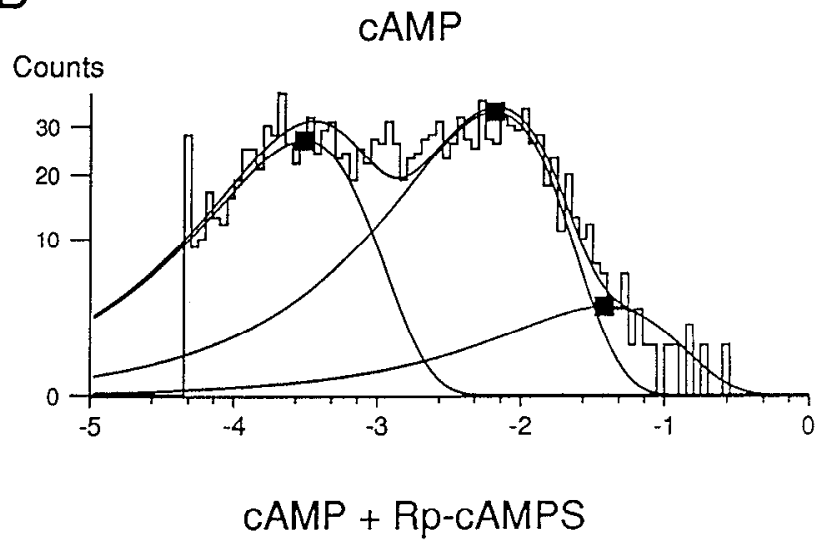

Counts

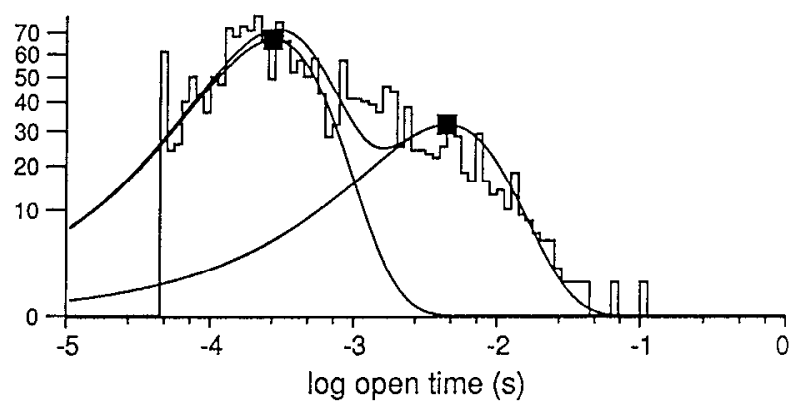

Figure 5. Effect of Rp-cAMPS on single CNG channels. $A$, Representative openings of a single OLF channel activated by $50 \mu \mathrm{M} c A M P$ in the absence and presence of $500 \mu \mathrm{M}$ Rp-cAMPS (cAMP $+R p-c A M P S)$ at $-100 \mathrm{mV} . B$, Open time histograms constructed from the same patch using a burst criterion of $1 \mathrm{msec}$ and corrected for missed events. Histograms containing 1400 and 2075 events were fit with either 3 (cAMP) or 2 (cAMP + Rp-cAMPS) exponentials, respectively. Time constants for each exponential are indicated by filled squares.

\section{Differential effects of the Rp-phosphorothioate derivatives of cGMP on olfactory and photoreceptor CNG channels}

Sp-cGMPS, a phosphorothioate derivative of cGMP, is an agonist of olfactory CNG channels, activating $80 \%$ of the maximal CNG current when applied at $3 \mathrm{~mm}$, without reducing the response to coapplied cGMP (data not shown). Previous studies also have shown that Sp-cGMPS is a full agonist of photoreceptor CNG channels (Zimmerman et al., 1.985).

Whereas the Sp isomer appears to be a full agonist for olfactory and photoreceptor CNG channels, the Rp isomer affects the two channels in opposite ways: photoreceptor CNG channels are activated and olfactory CNG channels are inhibited competitively. These experiments used cloned $\mathrm{CNG}$ channels expressed in Xenopus oocytes: OLF channels derived from catfish olfactory neurons, and RET CNG channcls derived from bovine rod photoreceptors. RET channels are activated by both Rp-cGMPS and Rp-8-pCPT-cGMPS, and neither compound antagonizes activation of the channels elicited by $25 \mu \mathrm{M}$ cGMP. In fact, Rp-cGMPS is a low-affinity agonist of RET channels, with $1 \mathrm{~mm}$ activating 35 $\pm 6 \%(n=3)$ of the maximal CNG current. This value is consistent with the findings of Zimmerman et al. (1985), who showed that Rp-cGMPS aclivates native rod CNG channels with a $K_{1 / 2}$ of $\sim 1200 \mu \mathrm{M}$. Much lower concentrations of Rp-8-pCPTcGMPS are required to activate the RET channels (e.g., $10 \mu \mathrm{M}$ ). However, at high concentrations (e.g., $100 \mu \mathrm{M}$ ), Rp-8-pCPTcGMPS apparently causes open-channel block of the RET channel that obscures observation of full activation of the current. The blocking effect is voltage-dependent, suggesting that Rp-8-pCPT-
cGMPS crosses part of the membrane electrical field and occludes the pore. At highly depolarized potentials $(+120 \mathrm{mV})$, the block is largely relieved, and the maximal RET CNG current activated by Rp-8-pCPT-cGMPS is estimated to be $93 \%$ of the maximal cGMP response, with an estimated $K_{1 / 2}$ of $11 \mu \mathrm{M}$ (Fig. $6 A$ ).

In contrast to RET channels, OLF channels exhibit little activation in response to $500 \mu \mathrm{M}$ Rp-cGMPS $(<2 \% ; n=3)$. Rp-8-pCPT-cGMPS is only slightly more effective, activating only $4-9 \%$ of the maximal CNG current (Fig. $6 B$ ). Despite its low efficacy, Rp-8-pCPT-cGMPS does bind to the OLF channel, since $500 \mu \mathrm{M}$ strongly antagonizes cGMP activation of OLF channels (Fig. 6B). The inhibition produced by Rp-8-pCPTcGMPS is competitive; it blocks activation of $\mathrm{CNG}$ current elicited by $25-100 \mu \mathrm{M}$ cGMP completely, and inhibits a progressively smaller percentage of the CNG current elicited by 1-10 mM cGMP. The compctitive nature of the inhibition caused by Rp-8-pCPT-cGMPS suggests that it binds to the same site as does cGMP and demonstrates that direct block of the channel is not a major contributor to inhibition of the OLF channel.

\section{Molecular determinant of agonist versus antagonist action elicited by Rp-8-pCPT-cGMPS}

The observation that Rp-8-pCPT-cGMPS is an agonist for the RET channel and an antagonist for the OLF channel suggests that even though the two channels bind the ligand, they differ in a protein region that couples binding to channel gating. To identify this region, we examined chimeric combinations between RET and OLF channels constructed previously to identify regions un- 
A

\section{Activation of RET}

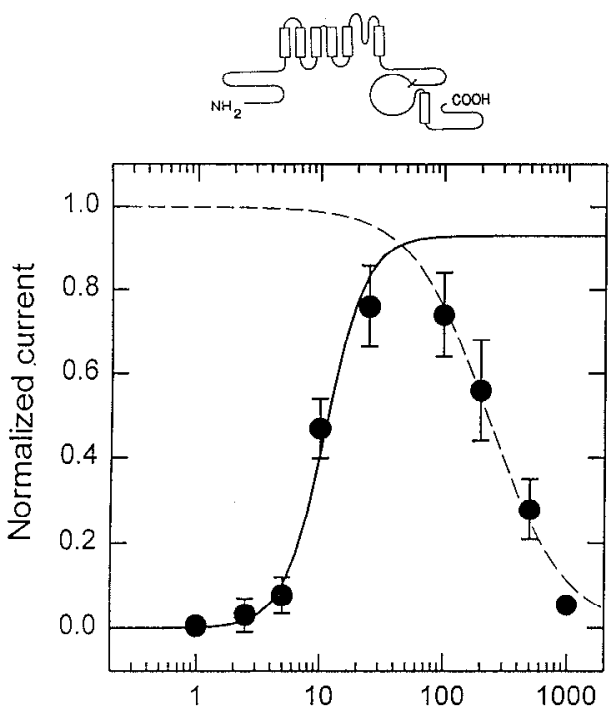

Rp-8-pCPT-cGMPS concentration $(\mu \mathrm{M})$
B

\section{Antagonism of OLF}

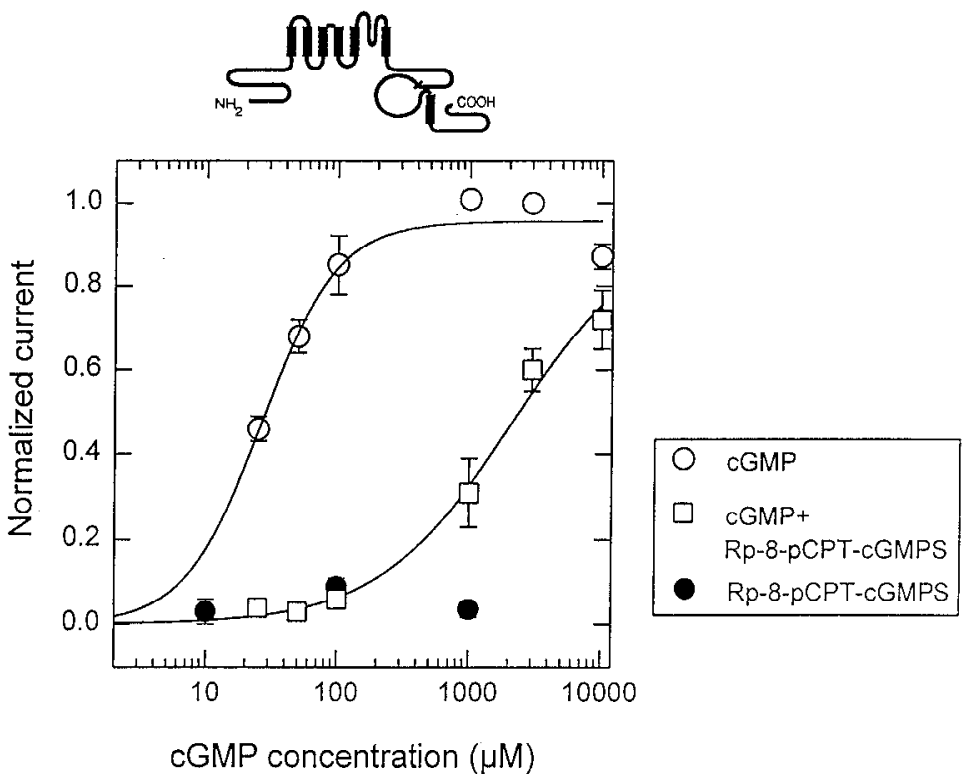

Figure 6. Differential effect of Rp-8-pCPT-cGMPS on RET and OLF. A, Effect of Rp-8-pCPT-cGMPS on RET channels (N $=4$ patches). Curves show fil to the Hill equation of activation (solid line; $K_{1 / 2}=11 \mu \mathrm{M}$ ) and block (dotled line; $K_{1 / 2}-228 \mu \mathrm{M}$ ). The amount of voltage-dependent block was minimized by measuring currents at the end of $750 \mathrm{msec}$ pulses to $+120 \mathrm{mV}$. $B$, Dose-response curves showing antagonism of OLF channels. Data from 4-9 experiments show effect of cGMP alone (open circles) and cGMP in the presence of $500 \mu \mathrm{M}$ Rp-8-pCPT-cGMPS (open squares). Solid curves show fits to the Hill equation with $K_{1 / 2}=26.6$ and $2150 \mu \mathrm{M}$, respectively. Application of 10,100 , and $1000 \mu \mathrm{M}$ Kp-8-pCPT-cGMPS alone activated 3, 9, and $4 \%$ of the maximal CNG current (filled circles), respectively. All currents were normalized to responses elicited by 1-10 mM cGMP.

derlying the different responses of RET and OLF channels to cAMP and cGMP (Goulding et al., 1994). This previous study found that two domains account for the differences in channel activation by cAMP and cGMP: one domain consists of the $\mathrm{N}$ terminus and first two membrane-spanning segments ( $\mathrm{N}-\mathrm{S} 2$ domain); the second domain is the conserved cyclic nucleotidebinding region in the $\mathrm{C}$ terminus of the protein.

To test the role of the $\mathrm{C}$ terminus in determining the response to Kp-8-pCPT-cGMPS, we examined ROC, the RET channel with a substituted OLF C terminus, and ORC, the OLF channel with a substituted RET $C$ terminus. Figure 7 shows the response of these chimeras to Rp-8-pCPT-cGMPS. As noted above, Rp-8pCPT-cGMPS activates RET and fails to antagonize the response elicited by cGMP, whereas with OLF, Rp-8-pCPT-cGMPS antagonizes cGMP activation competitively and elicits very little current on its own. ORC behaves similarly to RET; Rp-8-pCPTcGMPS activates the CNG current fully and fails to antagonize the cGMP response (Fig. 7A). Likewise, ROC behaves like OLF; Rp-8-pCPT-cGMPS activates little current and strongly antagonizes activation by cGMP in an apparently competitive manner (Fig. $7 B$ ). Hence, the C-terminal domain alone can account for the difference in the actions of Rp-8-pCPT-cGMPS on the RET and OLF channels.

Does the N-S2 domain also contribute to determining whether Rp-8-pCPT-cGMPS is an agonist or antagonist? To address this question, we examined RON-S2, a chimera consisting of the RET channel with a substituted OLF N S2 domain (Fig. 7C). Like RET, RON-S2 is activated fully by Rp-8-pCPT-cGMPS, and no antagonism of cGMP action is evident. In fact, Rp-8-pCPTcGMPS activates RON-S2 with a 10-fold higher apparent affinity than it does for RET, and indeed Rp-cGMPS also can be ob- served to activate this channel fully. Hence, the N-S2 domain does not account for the differences in pharmacology of RET and OLF regarding Rp-8-pCPT-cGMPS and Rp-cGMPS.

$\Lambda$ additional chimeric channel enabled us to map the molecular determinant of Rp-cGMPS action to the conserved cyclic nucleotide-binding domain within the C-terminal domain. Recent studies have shown that gating of the bovine rod channel and the rat olfactory channel can be modulated when $\mathrm{Ni}^{2+}$ ions bind to specific histidine residues located in the cytoplasmic $\mathrm{C}$ terminus just beyond the last transmembrane domain, but outside of the putative cyclic nucleotide-binding site (Gordon and Lagotta, 1995a,b). These studies suggest that this region plays an important role in regulating channel gating and may determine the efficacy of CNG channels to different cyclic nucleotides. To test whether this region, or the conserved cyclic nucleotide-binding domain, contains the determinant for Rp-8-pCPT-cGMPS action, we generated a new chimera that contains both the $\mathrm{N}-\mathrm{S} 2$ domain and the conserved cyclic nucleotide-binding domain of the OLF channel, but is otherwise identical to RET. This chimeric channel, RON$\mathrm{S} 2-\mathrm{BD}$, demonstrates that the conserved cyclic nucleotide-binding domain indeed is the crucial site for determining Rp-8-pCPTcGMPS action. In contrast to RON-S2, where Rp-8-pCPTcGMPS is a full agonist, Rp-8-pCPT-cGMPS elicits remarkably littlc activation of CNG current in RON-S2-BD channels $(0.2 \%$ of the maximal current at $1 \mathrm{~mm} ; n=3$ ). However, Rp-8-pCPTcGMPS does bind to RON-S2-BD channels. Figure $7 D$ shows that Rp-8-pCPT-cGMPS fully antagonizes CNG current elicited by cGMP in these channels, exhibiting an $\mathrm{IC}_{50}$ of $51 \mu \mathrm{M}$. The inhibition was equally effective at $+80 \mathrm{mV}$ as at $-80 \mathrm{mV}$, establishing that voltage-dependent block was not involved. Rp-cGMPS (1 


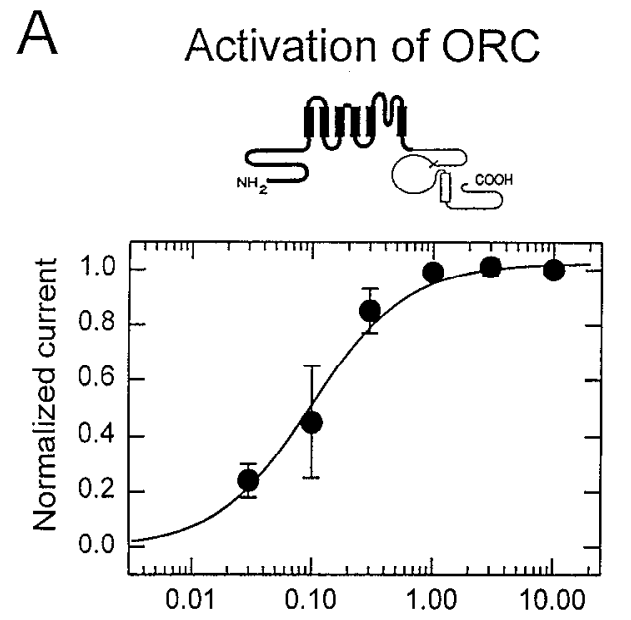

Rp-8-pCPT-cGMPS concentration ( $\mu \mathrm{M})$

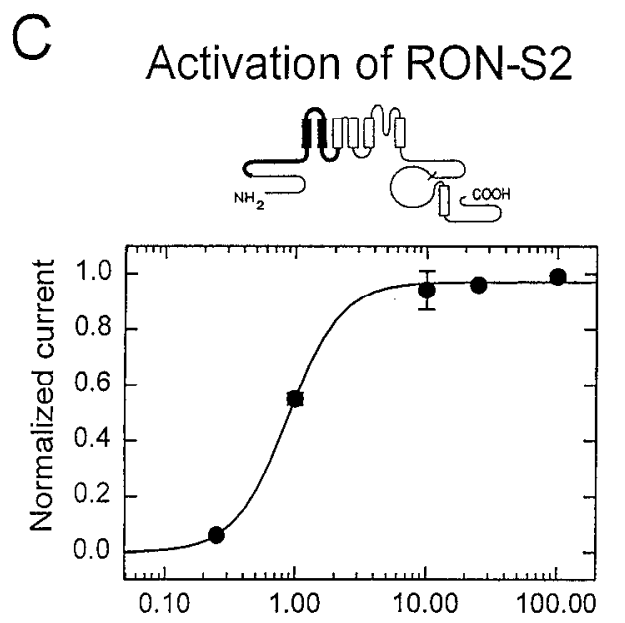

Rp-8-pCPT-cGMPS concentration ( $\mu \mathrm{M})$
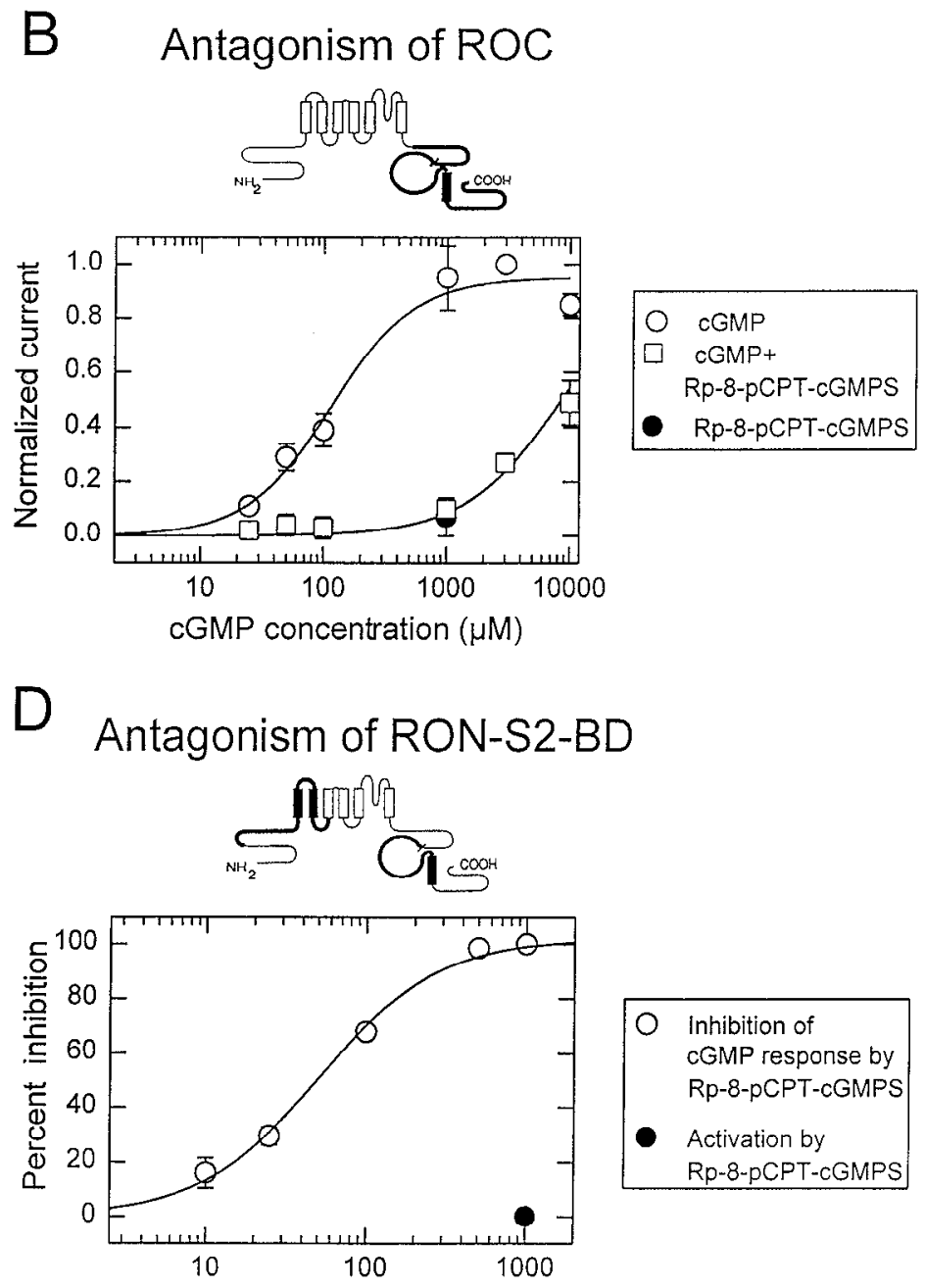

Rp-8-pCPT-cGMPS concentration $(\mu \mathrm{M})$

Figure 7. The molecular determinant for Rp-8-pCPT-cGMPS action on CNG channels. A, Full activation of ORC channels by Rp-8-pCPT-cGMPS. Concentrations of Rp-8-pCPT-cGMPS up to $1 \mathrm{~mm}$ continued to activate CNG current fully (data not shown). Curve shows Hill fit with $K_{1 / 2}, n$, and $I_{\text {max }}$ of $0.10 \mu \mathrm{M}, 1.1$, and 1.03, respectively $(N=3-6$ patches). $B$, Antagonism of ROC channels by Rp-8-pCPT-cGMPS. Mean data from three experiments show the effect of cGMP alone (open circles) and cGMP in the presence of $500 \mu \mathrm{M}$ Rp-8-pCPT-cGMPS (open squares). Curves show IIill fits with $K_{1 / 2}$ $=115$ and $8700 \mu \mathrm{M}$, respectively. Application of $1 \mathrm{~mm}$ Rp-8-pCPT-cGMPS alone activated $7.0 \pm 7.0 \%$ of the maximal CNG current (filled circles). $C$, Full activation of RON-S2 channels by Rp-8-pCPT-cGMPS. Curve shows Hill fit to the data with $K_{1 / 2}, n$, and $I_{\max }$ of $71 \mu \mathrm{M}, 2.1$, and 0.98 , respectively $(N=4$ patches). $D$, Antagonism of RON-S2-BD channels by Rp-8-pCPT-cGMPS. Inhibition of responses to half-saturating cGMP concentration (250 $\mu \mathrm{M})$ by different concentrations of Rp-8-pCPT-cGMPS. The estimated IC $\mathrm{I}_{50}$ was $51 \mu \mathrm{M}$. Data represent mean \pm SEM for three measurements. Application of $1 \mathrm{~mm}$ Rp-8-pCPT-cGMPS alone activated $0.2 \pm 0.1 \%$ of the maximal CNG current $(N=2$ patches).

mM) also antagonized cGMP-activated current by $\sim 30 \%$ while eliciting no activation of the channels.

\section{DISCUSSION}

Our results show that phosphorothioate derivatives of cAMP and cGMP have different actions on photoreceptor and olfactory CNG channels. Table 1 summarizes these effects on cloned versions of the channels (RET and OLF). The phosphorothioate ligand molecules differ both in their purine rings (adenine in cAMP vs guanine in CGMP) and in the position of the sulfur atom on the cyclized phosphate ( $\mathrm{S}$, or axial position, vs $\mathrm{R}$, or equatorial position). These two parts of the ligand apparently are recognized in different ways by the RET and OLF channels. With regard to gating, the OLF channel is indifferent to the nature of the purine ring, but instead discriminates between the $\mathrm{Rp}$ and $\mathrm{Sp}$ isomers, with the $\mathrm{Rp}$ isomers being antagonists and the $\mathrm{Sp}$ isomers being agonists. In contrast, the gating of RET channels does discriminate between purine rings, with
cGMP analogs being agonists and cAMP analogs being antagonists. Thus, although the putative binding sites of the CNG channels share considerable sequence homology, they differ in the way that ligand binding is coupled to channel gating.

Table 1 also shows that Rp-cGMPS is an agonist for the RET channel and an antagonist for the OLF channel. Which parts of the proteins are responsible for this difference? Possible regions include all or part of the binding site on the C terminus, and the $\mathrm{N}-\mathrm{S} 2$ region, which has been implicated in mediating differences in the ability of cAMP and CGMP to activate the RET and OLF channels (Goulding et al., 1994). The N-S2 domain does not appear to be responsible, because introduction of the OLF $\mathrm{N}-\mathrm{S} 2$ region into RET does not transform the pharmacological properties of the channel, but instead makes Rp-cGMPS and its 8-pCPT derivative even more potent agonists. Rather, our experiments with the ROC and ORC chimeras show that the relevant part of the CNG channel protein for deter- 
Table 1. Summary of effects of cyclic nucleotides and phosphorothioate derivatives on $\mathrm{CNG}$ channels

$\mathrm{OLF}^{a}$

RET $^{a}$

\begin{tabular}{lll}
\hline cAMP & Agonist $^{b}$ & Weak antagonist $^{c}$ \\
Rp-cAMPS & Antagonist & Antagonist \\
Sp-cAMPS & Agonist & Weak antagonist ${ }^{e}$ \\
\hline cGMP & Agonist $^{b}$ & Agonist \\
Rp-cGMPS & Antagonist $^{f}$ & Agonist \\
Sp-cGMPS & Agonist $^{b}$ & Agonist $^{h}$ \\
\hline
\end{tabular}

${ }^{a}$ Actions of cyclic nucleotides on OLF and RET CNG channels expressed in Xenopus oocytes, except where noted otherwise. Lack of agonist activity is defined as activation of $<5 \%$ of the maximal current at saturating concentration of ligand. Maximal CNG current is defined as the current elicited by [cGMP] $\geq 1 \mathrm{mM}$. All 8-pCPT derivatives have an equivalent pharmacology to substituted cyclic nucleotides but have higher apparent affinities.

${ }^{b}$ See Goulding et al. (1994)

${ }^{c}$ cAMP activates $2 \%$ of the maximal RET CNG current (Groulding et al., 1994). Saturating cAMP activates $25 \%$ of the native rod $\mathrm{CNG}$ current and antagonizes activation by cGMP (Furman and Tanaka, 1989).

${ }^{d}$ Rp-cAMPS activates $2 \%$ of the maximal OLF CNG current and $34 \%$ of the native olfactory $\mathrm{CNG}$ current at saturation.

$e 1 \mathrm{~mm}$ elicits no detcctable responsc and reduccs current clicitcd by $25 \mu \mathrm{M}$ cGMP by $30 \%$.

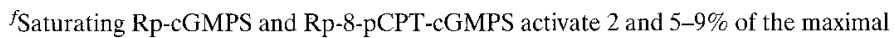
OLF CNG current, respectively.

$83 \mathrm{mM}$ Sp-cGMPS activates $80 \%$ of the maximal OLF CNG current, whereas antagonism of the cGMP response is not detected.

${ }^{h} 1 \mathrm{~mm}$ activates $32 \%$ of the maximal $\mathrm{CNG}$ current, and antagonism is not detected. In native rod CNG channels, $k_{1 / 2}=210 \mu \mathrm{M}$ (Zimmerman et al., 1985).

mining agonism versus antagonism is the $C$ terminus. Comparison of RON-S2 and RON-S2-BD further narrows the localization of the crucial site to the 125-amino-acid putative cyclic nucleotide-binding domain. Hence, Rp-8-pCPT-cGMPS is a full agonist on RON-S2 and a nearly pure antagonist on RON-S2-BD. The 125-amino-acid region is still rather broadly defined. To pinpoint specific amino acid differences that account for the functional differences in phosphorothioate action, additional chimeras and point mutants will need to be constructed and tested.

The putative cyclic nucleotide-binding site shares sequence homology with catabolite-gene activator protein (CAP), a bacterial cAMP-binding protein (Altenhofen et al., 1991; Goulding et al., 1994). X-ray crystallography of CAP has shown that its cAMPbinding domain is composed of three $\alpha$-helices $(\mathrm{A}, \mathrm{B}$, and $\mathrm{C}$ ) and an eight-stranded $\beta$-barrel structure (Weber and Steitz, 1987; Shabb and Corbin, 1992). The purine ring of cAMP contacts residues in the $\mathrm{C} \alpha$-helix, whereas the cyclized phosphate contacts residues in the $\beta$-barrel. Because the RET and OLF channels choose which ligand is an agonist or antagonist based on different parts of the cyclic nucleotide molecule, the part of the protein that discriminates between agonist and antagonist may differ between RET and OLF. The OLF channel discriminates between agonist and antagonist based on the phosphorothioate group; hence, the region that determines whether this channel will gate may be located in a region homologous to the $\beta$-barrel. Likewise, because the RET channel discriminates between agonist and antagonist based on the purine ring, a region homologous to the $\mathrm{C} \alpha$-helix also may be responsible in this channel. This hypothesis may be tested by generation of additional constructs.

The specific amino acids that determine whether Rp-cGMPS and its pCPT derivative are agonists or antagonists are likely to be different from those identified previously as crucial for binding the phosphate and for mediating activation in both CAP and in the cyclic nucleotide-dependent protein kinases. Rp-cAMPS strongly activates CAP, whereas Sp-cAMPS is a weak activator (Scholubbers et al., 1984). Two amino acids, $\mathrm{Arg}^{82}$ and $\mathrm{Ser}^{83}$, are thought to interact with the phosphate and determine the chiral specificity of CAP activation (Weber and Steitz, 1987). Amino acids in positions equivalent to $\mathrm{Arg}^{82}$ and $\mathrm{Ser}^{83}$ in PKA and PKG likewise are crucial for activation by cAMP and cGMP (Weber et al., 1989) and determine whether Rp-cAMPS acts as an agonist or an antagonist in PKA (Dostmann and Taylor, 1991). These putative contact sites with cyclic nucleotides also are highly conserved in the CNG channcls ("RTAN" sequence in the $\beta 7$ domain) (Goulding et al., 1994), and the specific nature of the amino acids also is crucial for activation (Altenhofen et al., 1991). However, because the sequences of RET and OLF are identical in this region, this cannot be the site responsible for functional differences between the two channels.

The chimeric substitutions that convert the Rp-cGMPS ligands from agonists to antagonists also cause a decrease in the apparent affinity for cGMP itself, suggesting that the coupling between binding and gating has been weakened generally in these channels. Is the shift from agonist to antagonist as the putative binding domain is converted from RET to OLF caused by a general change in the ability of ligands to activate the channel? Indeed, in a variety of CNG channel chimeras there is a correlated change in the ability of cAMP and cGMP to gate the channels, caused by similar shifts in the free energy changes when each of the cyclic nucleotides open the channel (Goulding et al., 1994; Gordon and Zagotta, 1995b). However, simply decreasing the ease of activation cannot explain why Rp-8-pCPT-cGMPS is converted from an agonist to an antagonist in RON-S2 versus RON-S2-BD. Rp-8pCPT-cGMPS is a full agonist of higher affinity than cGMP for RON-S2 $\left(K_{1 / 2}\right.$ values are 1 and $4 \mu \mathrm{M}$, respectively; Fig. $7 C$ ) (Goulding et al., 1994). Therefore, decreasing the ability to open when the binding site is saturated would reduce the effectiveness of cGMP at least as much as it would Rp-8-pCPT-cGMPS. However, the effect of Rp-8-pCPT-cGMPS is transformed selectively in RON-S2-BD. In RON-S2-BD, the analog is almost completely ineffective at opening the channel. However, it still has a higher apparent affinity than cGMP for binding to the channel $\left(\mathrm{IC}_{50}\right.$ of $51 \mu \mathrm{M}, K_{1 / 2}$ of $310 \mu \mathrm{M}$, respectively; Fig. $7 D$ ) (our unpublished observations). Hence, the putative binding domain must contain regions that determines selectively whether these phosphorothioate derivatives are agonists or antagonists in addition to residues that determine the effectiveness of cGMP and cAMP.

In addition to their usefulness in probing the molecular basis of CNG channel activation, the phosphorothioate antagonists of $\mathrm{CNG}$ channels will be useful for investigating the functions of CNG channels in intact cells. These compounds will be useful for classifying CNG channels in other cell types as OLF-like or RETlike, or those having a novel pharmacological profile. A possible complicating factor is that recent studies show that CNG channels in photoreceptors (Chen et al., 1994) and olfactory receptor neurons (Bradley et al., 1994; Liman and Buck, 1994) are heteromeric, containing $\beta$-subunits in addition to the original $\alpha$-subunits (e.g., RET and OLF). Although the native $\mathrm{CNG}$ channels differ from the cloned CNG channels in having higher sensitivities to cAMP and cGMP, our results show that qualitatively, the pharmacological profiles of the native channels are nearly identical to those of cloned RET and OLF channels. Keeping in mind their effects on cyclic nucleotide-dependent protein kinases and PDEs, the phosphorothioate cyclic nucleotide derivatives thus can be added to the arsenal of agents used to implicate $\mathrm{CNG}$ channels in physio- 
logical responses. In particular, the membrane-permeant pCPT derivatives of Rp-cAMPS and Rp-cGMPS are effective antagonists of CNG channels and will compliment the use of agonists in identifying the role of CNG channels in intact cells.

\section{REFERENCES}

Ahmad I, Leinders-Zufall T, Kocsis JD, Shepherd GM, Zufall F, Barnstable CJ (1994) Retinal ganglion cells express a cGMP-gated cation conductance activatable by nitric oxide donors. Neuron 12:155-165.

Altenhofen W, Ludwig J, Eismann E, Kraus W, Bonigk W, Kaupp UB (1991) Control of ligand specificity in cyclic nucleotide-gated channels from rod photoreceptors and olfactory epithelium. Proc Natl Acad Sci USA 88:9868-9872.

Biel M, Zong X, Distler M, Bosse E, Klugbauer N, Murakami M, Flockerzi V, Hofmann F (1994) Another member of the cyclic nucleotidegated channel family expressed in testis, kidney, and heart. Proc Natl Acad Sci USA 91:3505-3509.

Botelho LHP, Rothermael JD, Coombs RV, Jastorff B (1988) cAMP analog antagonists of cAMP action. Methods Enzymol 159:159-172.

Bradley J, Li J, Davidson N, Lester HA, Zinn K (1994) Heteromeric olfactory cyclic nucleotide-gated channels: a subunit that confers increased sensitivity to cAMP. Proc Natl Acad Sci USA 91:8890-8894.

Brown RL, Bert RJ, Evans FE, Karpen JW (1993) Activation of retinal rod cGMP-gated channels: what makes for an effective 8-substituted derivative of cGMP? Biochemistry 32:10089-10095.

Butt E, Van Bemmelen M, Fischer L, Walter U, Jastorff B (1990) Inhibition of cGMP-dependent protein kinase by Rp-guanosine $3^{\prime}, 5^{\prime}$ monophosphorothioate. FEBS Lett 263:47-50.

Chen T-Y, Peng Y-W, Dhallan RS, Ahamed B, Reed RR, Yau K-W (1993) A new subunit of the cyclic nucleotide-gated cation channel in retinal rods. Nature 362:764-767.

Dhallan RS, Yau K-W, Schrader K, Reed R (1990) Primary structure and functional expression of a cyclic nucleotide-activated channel from olfactory neurons. Nature 347:184-187.

DiFrancesco D, Tortora P (1991) Direct activation of cardiac pacemaker channels by intracellular cyclic AMP. Nature 351:145 147.

Dostmann WRG, Taylor SS (1991) Identifying the molecular switches that determine $\left(R_{p}\right)$-cAMPS functions as an antagonist in the activation of cAMP-dependent protein kinase I. Biochemistry 30:8710-8716.

Fesenko EE, Kolesnikov SS, Lyubarsky AL (1985) Induction by cyclic GMP of a cationic conductance in plasma membrane of retinal rod outer segment. Nature 313:310-313.

Furman RE, Tanaka JC (1989) Photoreceptor channel activation: interaction between cAMP and cGMP. Biochemistry 28:2785-2788.

Gordon SE, Zagotta WN (1995a) A histidine associated with the gate of the cyclic nucleotide-activated channels in rod photoreceptors. Neuron $14: 177-183$.

Gordon SE, Zagotta WN (1995b) Localization of regions affecting an allosteric transition in cyclic nucleotide-activated channels. Neuron $14: 857-864$.
Gordon SE, Brautigan DL, Zimmerman AL (1992) Protein phosphatases modulate the apparent agonist affinity of the light-regulated ion channel in retinal rods. Neuron 9:739-748.

Goulding EH, Ngai J, Kramer RH, Colicos S, Axel R, Siegelbaum SA, Chess A (1992) Molecular cloning and single channel properties of the cyclic nucleotide-gated channel from cattish olfactory neurons. Neuron 8:45-58.

Goulding EH, Tibbs G, Siegelbaum SA (1994) Molecular mechanism of cyclic-nucleotide-gated channel activation. Nature 372:369-374.

Kaupp BU, Niidome T, Tanabe T, Terada S, Bonigk W, Stuhmer W, Cook NJ, Kangawa K, Matsuo H, Hirode T, Miyata T, Numa S (1989) Primary structurc and functional expression from complementary DNA of the rod photoreceptor cGMP-gated channel. Nature 342:762-766.

Kramer RH, Siegelbaum SA (1992) Intracellular $\mathrm{Ca}^{2+}$ regulates the sensitivity of cyclic nucleotide-gated channels in olfactory receptor neurons. Neuron 9:897-906.

Liman ER, Buck LB (1994) A second subunit of the olfactory cyclic nucleotide-gated channel confers high sensitivity to cAMP. Neuron 13:611-621.

Matthews G, Watanabe S (1987) Properties of ion channels closed by light and opened by guanosine $3^{\prime}, 5^{\prime}$-cyclic monophosphate in toad retinal rods. J Physiol (Lond) 389:691-715.

Nakamura T, Gold GH (1987) A cyclic nucleotide-gated conductance in olfactory receptor cilia. Nature 325:442-444.

Nawy S, Jahr CE (1991) cGMP-gated conductance in retinal bipolar cells is suppressed by the photoreceptor neurotransmitter. Neuron 7:677-683.

Scholubbers HG, van Knippenburg PH, Baraniak J, Stec WJ, Morr M, Jastorff B (1984) Investigations on stimulation of lac transcription in vivo in Escherichia coli by cAMP analogues: biological activities and structure-activity correlations. Eur J Biochem 138:101-109.

Shabb JM, Corbin JD (1992) Cyclic nucleotide-binding domains in proteins having diverse functions. J Biol Chem 267:5723-5726.

Weher IT, Steitz TA (1987) Structure of a complex of cataholite gene activator protein and cyclic AMP refined at $2.5 \AA$ resolution. J Mol Biol 198:311-326.

Weber IT, Shabb JB, Corbin JD (1989) Predicted structure of the cGMP binding domains of the cGMP-dependent protein kinases: a key alanine/threonine difference in evolutionary divergence of CAMP and cGMP binding siles. Biochemistry 28:6122-6127.

Weyand I, Godde M, Frings S, Weiner J, Muller F, Altenhofen W, Hatt H, Kaupp UB (1994) Cloning and functional expression of a cyclic nucleotide-gated channel from mammalian sperm. Nature 368:859-863.

Yau K-W (1994) Cyclic nucleotide-gated channels: an expanding new family of ion channels. Proc Natl Acad Sci USA 91:3481-3483.

Zimmerman AL, Yamanaka G, Eckstein F, Baylor DA, Stryer L (1985) Interaction of hydrolysis-resistant analogs of cyclic GMP with the phosphodiesterase and light-sensitive channel of retinal rod outer segments. Proc Natl Acad Sci USA 82:8813-8817.

Zimmerman AL, Karpen JW, Baylor DA (1988) Hindered diffusion in excised patches from retinal rod outer segments. Biophys J 54:351-355. 